Check for updates

Cite this: Chem. Commun., 2017, 53, 9238

Received 28th June 2017, Accepted 27th July 2017

DOI: $10.1039 / c 7 c c 04977 c$

rsc.li/chemcomm

\section{An atypical interaction explains the high-affinity of a non-hydrolyzable S-linked 1,6- $\alpha$-mannanase inhibitor}

\author{
Tyson Belz, ${ }^{a}$ Yi Jin, ${ }^{b}$ Joan Coines, ${ }^{c}$ Carme Rovira, (D)*cd Gideon J. Davies (D) ${ }^{\mathrm{b}}$ and \\ Spencer J. Williams (D) *a
}

\begin{abstract}
The non-hydrolyzable S-linked azasugars, 1,6- $\alpha$-mannosylthio- and 1,6- $\alpha$-mannobiosylthioisofagomine, were synthesized and shown to bind with high affinity to a family 76 endo-1,6- $\alpha$-mannanase from Bacillus circulans. X-ray crystallography showed an atypical interaction of the isofagomine nitrogen with the catalytic acid/base. Molecular dynamics simulations reveal that the atypical binding results from sulfur perturbing the most stable form away from the nucleophile interaction preferred for the O-linked congener.
\end{abstract}

1,6- $\alpha$-Mannans are polysaccharides produced by fungi and bacteria., ${ }^{1,2}$ In the fungal glycocalyx, such as that of the ascomycetes Saccharomyces cerevisiae and Candida albicans, they are present as part of the outer chain mannan, ${ }^{1}$ and are especially abundant in the latter organism, comprising up to $40 \%$ of the mass of the cell wall. ${ }^{3}$ Within bacteria, 1,6- $\alpha$-mannans are found within the lipomannans and lipoarabinomannans of actinomycetes such as mycobacteria and corynebacteria. ${ }^{4}$ 1,6- $\alpha$-Mannans are degraded by endo-1,6- $\alpha$-mannanases, enzymes that cleave the polysaccharide chains into smaller fragments that are then further degraded by exo-acting mannosidases. All known 1,6- $\alpha$-mannanases fall into family 76 of the glycoside hydrolase $(\mathrm{GH})$ classification scheme (www.cazy.org; www.cazypedia.org), ${ }^{5}$ which also includes putative fungal transglycosidases that have been proposed to act on protein glycosylphosphatidylinositol anchors. ${ }^{1,6}$ The gut microbiota resident Bacteroides thetaiotaomicron produces GH76 endo-1,6$\alpha$-mannanases as cell surface and periplasmic enzymes that enable the depolymerisation of fungal cell wall mannan

\footnotetext{
${ }^{a}$ School of Chemistry and Bio21 Molecular Science and Biotechnology Institute, University of Melbourne, Parkville, Victoria 3010, Australia.

E-mail: sjwill@unimelb.edu.au

${ }^{b}$ York Structural Biology Laboratory, Department of Chemistry, University of York, Heslington, YO10 5DD, UK.E-mail: gideon.davies@york.ac.uk

${ }^{c}$ Departament de Química Inorgànica i Orgànica (Secció de Química Orgànica) \& Institut de Química Teórica i Computacional (IQTCUB), Universitat de Barcelona, 08028 Barcelona, Spain.E-mail: c.rovira@ub.edu

${ }^{d}$ Institució Catalana de Recerca i Estudis Avançats (ICREA), 08010 Barcelona, Spain

$\dagger$ Electronic supplementary information (ESI) available. See DOI: 10.1039/c7cc04977c
}
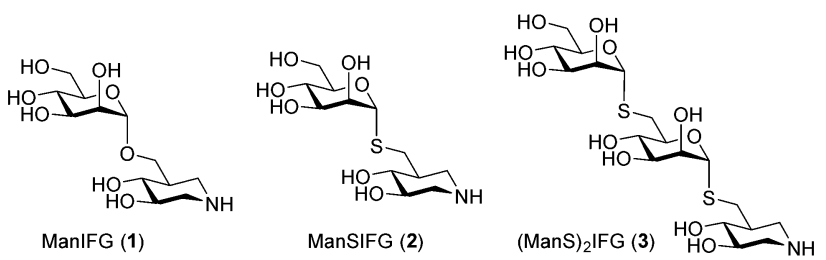

Fig. 1 Structures of S-linked and O-linked isofagomine-based inhibitors of Bacillus circulans endo-1,6- $\alpha$-mannanase (BcGH76).

consumed in the human diet. $^{7} \mathrm{GH}$ family 76 enzymes are also deployed by the soil bacterium Bacillus circulans, ${ }^{8}$ possibly to enable nutrient acquisition from fungal or actinomycete cell wall fragments. We recently reported the first inhibitor of a $\mathrm{GH}$ family 76 enzyme, ManIFG 1, which bound to the endo-1,6- $\alpha$ mannanase from $B$. circulans $(B c \mathrm{GH} 76)$ with modest affinity $\left(K_{\mathrm{D}}\right.$ $1.1 \mu \mathrm{M}$ ) (Fig. 1). This compound proved a useful mechanistic probe, and supplied evidence supporting a boat conformation at the enzyme-catalyzed transition state, and a ${ }^{\mathrm{O}} S_{2} \rightarrow B_{2,5} \rightarrow{ }^{1} S_{5}$ conformation pathway for GH family 76 catalysis. ${ }^{9}$

The strategy used for the design of ManIFG $\mathbf{1}$ built upon a rich history of glycosidase inhibitor design. ${ }^{10}$ It is widely appreciated that exo-acting glycosidases are effectively inhibited by sugarshaped heterocycle 'warheads' containing basic nitrogen in place of $\mathrm{C} 1$ or $\mathrm{O} 5$, e.g. isofagomine (IFG) or deoxymannojirimycin (DMJ). However, such simple monosaccharide-mimicking structures are frequently weak inhibitors of endo-acting glycosidases, which have multiple substrate binding subsites. By adding sugar residues to the warhead to mimic the natural substrate, effective inhibition of endoglycosidases can often be obtained. However, as such compounds have glycosidic linkages within, and are by virtue of the design process mimics of the substrate, the potential exists that they may themselves be substrates for their target enzymes. Typically, such concerns are limited for disaccharidebased inhibitors, as disaccharides are rarely optimal substrates for endoglycosidases, but become more significant for trisaccharides and above. For example, cellotriose- and cellotetraose-like isofagomines were shown to be cleaved by cellulase Cel9A from 
Alicyclobacillus acidocaldarius. ${ }^{11}$ Additionally, the natural product pseudo-tetrasaccharide inhibitor acarbose undergoes cleavage (and transglycosidation) by amylases. ${ }^{12}$

The natural product chitinase inhibitor allosamidin, which contains an allosamizoline inhibitor warhead, linked to $\mathrm{N}$-acetyl$\beta$-allosamine residues, is a poor substrate for chitinases presumably by virtue of its mismatched sugar stereochemistry. ${ }^{13}$ An alternative design strategy to limit hydrolysis by the target enzyme is to attach sugar residues to the inhibitor warhead through nonhydrolyzable linkages. For example, a $C$-glycosidic linkage was used within a non-hydrolyzable epoxyalkyl $C$-glycoside inhibitor of an exoglycosidase ${ }^{14}$ and alkylation of isofagomine on nitrogen by a sugar residue provided a weak inhibitor of isoamylase. ${ }^{15}$ In a related vein, a thioamide derivative of a chitooligosaccharide linked to the warhead NAG-thiazoline was not a substrate for chitinases that utilize neighboring group participation by virtue of the poorer nucleophilicity of the thionyl group relative to a carbonyl group. ${ }^{16}$ Given the similarity in size and bonding geometry of sulfur and oxygen, but the lower basicity of the former, an obvious alternative could be to use sulfur in place of oxygen in an S-glycosidic linkage. S-Linked oligosaccharides have been widely employed as non-hydrolyzable mimics of glycosides, yet to our knowledge, no s-linked imino/ azasugar endoglycosidase inhibitors have been reported. Here we report the synthesis and characterization of S-linked di- and trisaccharide azasugar inhibitors ManSIFG 2 and (ManS) $)_{2}$ IFG 3 targeting the endo-1,6- $\alpha$-mannanase $B c \mathrm{GH} 76$.

We have previously reported the preparation of a family of $\alpha-1,6-S$-linked di-, tetra- and hexamannosides. ${ }^{17}$ The key element of this approach was the use of $\alpha$-mannosyl thioacetates or isothiuronium bromides with defined anomeric stereochemistry as precursors of thiolate nucleophiles that can be used for substitution reactions of 6-deoxy-6-iodosugars. Accordingly, we undertook the synthesis of the protected 6-iodo-isofagomine 5, from isofagomine tartrate. The advanced intermediate, alcohol $\mathbf{4},{ }^{9}$ was iodinated using the procedure of Garegg and Samuelsson $\left(\mathrm{I}_{2}, \mathrm{Ph}_{3} \mathrm{P}\right.$, imidazole) ${ }^{18}$ to afford iodide 5 (Scheme 1a). Nucleophilic substitution proceeded in good yield upon treatment of $\mathbf{5}$ with mannosyl thiuronium bromide $6^{19}$ in the presence of $\mathrm{Et}_{3} \mathrm{~N},{ }^{20}$ affording disaccharide 7 (Scheme 1b). Deprotection was achieved in two steps: Zemplén deacylation using $\mathrm{NaOMe}$ in $\mathrm{MeOH}$ afforded the hexaol 8; then removal of the Cbz group using TFA/anisole, followed by ion exchange chromatography, affording ManSIFG 2. Similarly, treatment of iodide 5 with disaccharide thioacetate $9^{17}$ and $\mathrm{Et}_{2} \mathrm{NH}^{21}$ afforded the trisaccharide 10 (Scheme 1c). Deprotection was achieved in a similar fashion affording (ManS) $)_{2}$ IFG 3.

The binding of ManSIFG 2 and (ManS) $)_{2}$ IFG 3 to $B c$ GH76 was quantified by isothermal titration calorimetry. ManSIFG bound with $K_{\mathrm{D}}=0.90 \pm 0.03 \mu \mathrm{M}$ and (ManS) $)_{2}$ IFG bound with $K_{\mathrm{D}}=24.7 \pm$ $7.9 \mathrm{nM}$. The affinity of ManSIFG is similar to the O-linked variant, ManIFG $1\left(K_{\mathrm{D}}=1.1 \mu \mathrm{M}\right)$, showing that the effect on affinity upon replacement of the glycosidic oxygen with sulfur is minimal. $(\mathrm{ManS})_{2}$ IFG bound approximately 37-fold more tightly than ManSIFG, suggesting that extension of the inhibitor with an additional sugar residue enables capture of additional binding energy.

To illuminate the binding mode of compounds 2 and 3 with $B c \mathrm{GH} 76$, we soaked these inhibitors into crystals of the enzyme

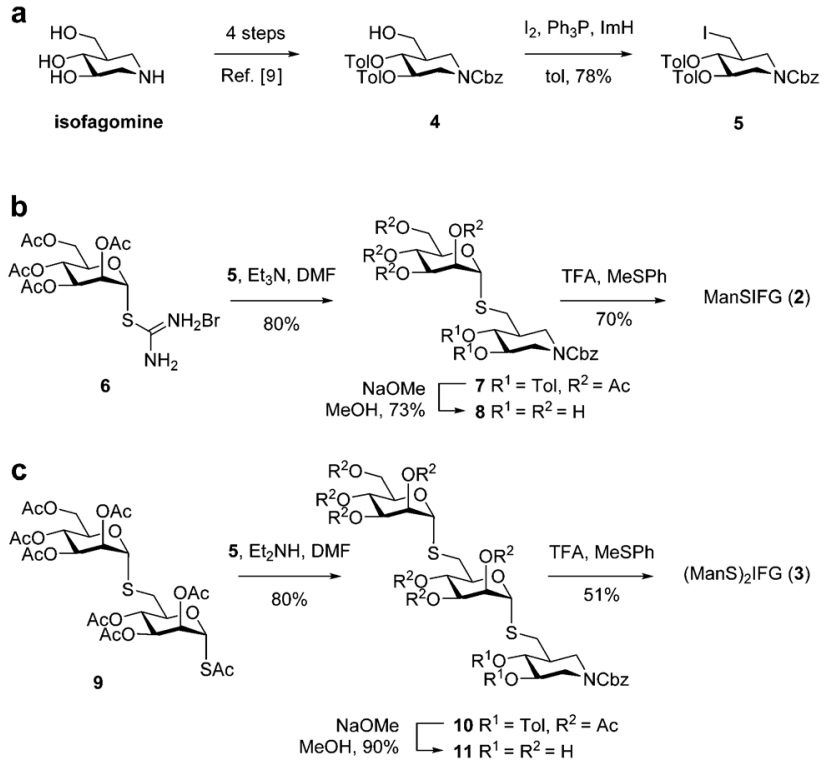

Scheme 1 Synthesis of S-linked isofagomine inhibitors ManSIFG (2) and (Man) ${ }_{2}$ SIFG (3). Tol = 4-methylbenzoyl.

and solved their X-ray structures to resolutions of 1.69 and $1.46 \AA$ A , respectively (Fig. $2 \mathrm{a}, \mathrm{b}$ and Table S1, ESI $\dagger$ ). ManSIFG 2 bound in the $-2 /-1$ subsites and (ManS) $)_{2}$ IFG 3 bound in the $-3 /-2 /-1$ subsites. As the interactions of the two inhibitors in the $-2 /-1$ subsites are essentially identical, the subsequent discussion will mainly focus on the higher affinity inhibitor (ManS) $)_{2}$ IFG 3. Superficially, the three residues of this inhibitor occupy similar positions to the equivalent residues in a previously reported Michaelis complex in which $\alpha$-mannopentaose was bound across the -4 to +1 subsites, and a complex with ManIFG $\mathbf{1}$, in which this inhibitor bound in the $-2 /-1$ subsites. ${ }^{9}$ In the $B c$ GH76-ManIFG complex the isofagomine moiety was in a $B_{2,5}$ conformation and engaged in a close contact $(2.8 \AA)$ with the enzymatic nucleophile, Asp124, an interaction that is typical for IFG-type inhibitors of retaining glycosidases (see summary in Table S2, ESI $\dagger$ ). Interestingly, a longer (and presumably weaker) interaction of ManIFG with the acid/base Asp125 was also evident $(\mathrm{N} \cdots \mathrm{O}=3.0 \AA)$. Close inspection of the complexes with compounds 2 and 3 reveals that while the non-reducing end residues are positioned in the -3 and -2 subsites in essentially identical fashions as for the previously reported complexes with O-linked substrates and inhibitors, the IFG head-groups are positioned atypically, such that the nitrogen is engaged in a close contact with the acid/base Asp125 (N. . O distance, $2: 2.7 \AA$, 3 : $2.6 \AA$ ) and an $\mathrm{N}-\mathrm{H} \pi$ interaction with Phe122 (for a summary of published complexes with IFG-type inhibitors see ESI, $\dagger$ Table S2). In contrast with the complex of ManIFG with $B c G H 76$, which was observed in a $B_{2,5}$ conformation, ${ }^{9}$ in these complexes the isofagomine moiety is found in an undistorted ${ }^{4} C_{1}$ conformation. ${ }^{22}$ We speculate that this close interaction involves a salt bridge with the protonated nitrogen of the inhibitor and the deprotonated Asp125 residue. Overlay of the binding mode of $\mathbf{1}$ and 2 or 3 , reveals that the binding of the latter pair of inhibitors results in a rotation of the IFG head group about the S-C4(IFG) bond (Fig. 2c). 
a

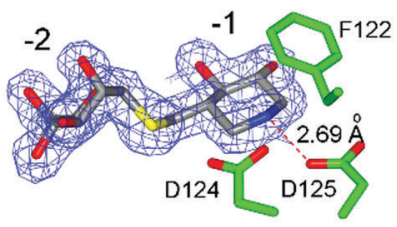

b

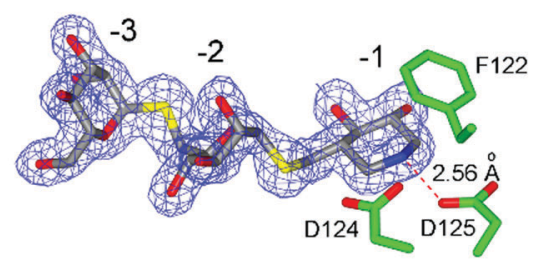

C

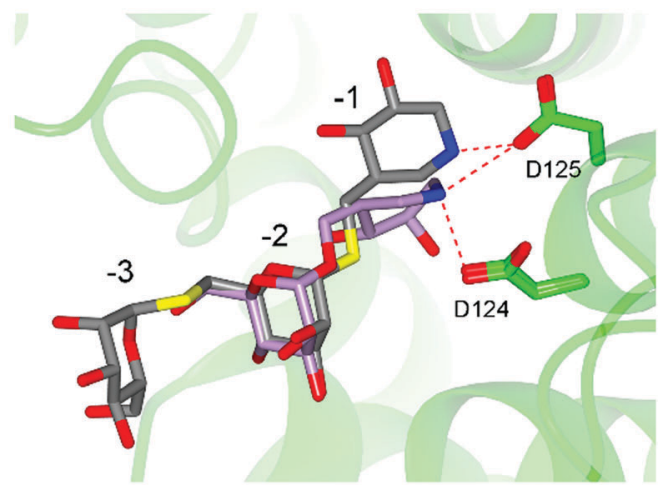

Fig. 2 Complexes of BcGH76 with (a) ManSIFG 2 (PDB: 5NOF) and (b) (ManS) ${ }_{2}$ IFG 3 (PDB: 5M77) (c) overlay of complexes of BcGH76 with $(\text { ManS) })_{2} \mathrm{IFG}$ and ManIFG 1 (PDB: 4D4D). ${ }^{9}$ Electron density meshes are $\sigma A$-weighted $2 F_{0}-F_{c}$ contoured at $1 \sigma\left(0.32\right.$ electrons per $\left.\AA^{3}\right)$, assembled using CCP4mg. ${ }^{23}$

While the binding of compounds 2 and 3 are atypical for IFG-based inhibitors in that they do not engage with the enzymatic nucleophile, the coincidentally similar affinities of compounds 1 and 2 most likely arise from the inhibitors achieving equivalent ionic interactions with the enzymatic acid/base. The present complexes merit comparison with the complex of a cellobiose-like isofagomine with the inverting GH family 9 Cel9A from Alicyclobacillus acidocaldarius. ${ }^{11}$ In that case two molecules of the cellobiose-like isofagomine were observed in the complex, with one bound in the typical $-2 /-1$ mode, with the isofagomine nitrogen interacting with the general acid residue (Asp146). Remarkably, a second molecule of cello-IFG was bound in a reversed mode in the $+2 /+1$ subsites, with the IFG moiety bound in the +1 subsite and the nitrogen interacting with the general base residue (Glu515).

We sought to understand the underlying reasons for the atypical binding modes observed for inhibitors 2 and 3 using a computational approach (molecular dynamics). ${ }^{24}$ Initially, we explored the protonation state of the catalytic residues in the $B c \mathrm{GH} 76-\mathrm{ManIFG}$ and $B c \mathrm{GH} 76-\mathrm{ManSIFG}$ complexes. In both cases, the IFG moiety was assumed to be protonated on the basis of its high basicity; this has been directly observed experimentally in crystallographic studies of cellulase Cel5A. ${ }^{25}$ Only models in which the ammonium-interacting carboxyl residue (the nucleophile and the acid/base, respectively) was deprotonated (Fig. 3 and
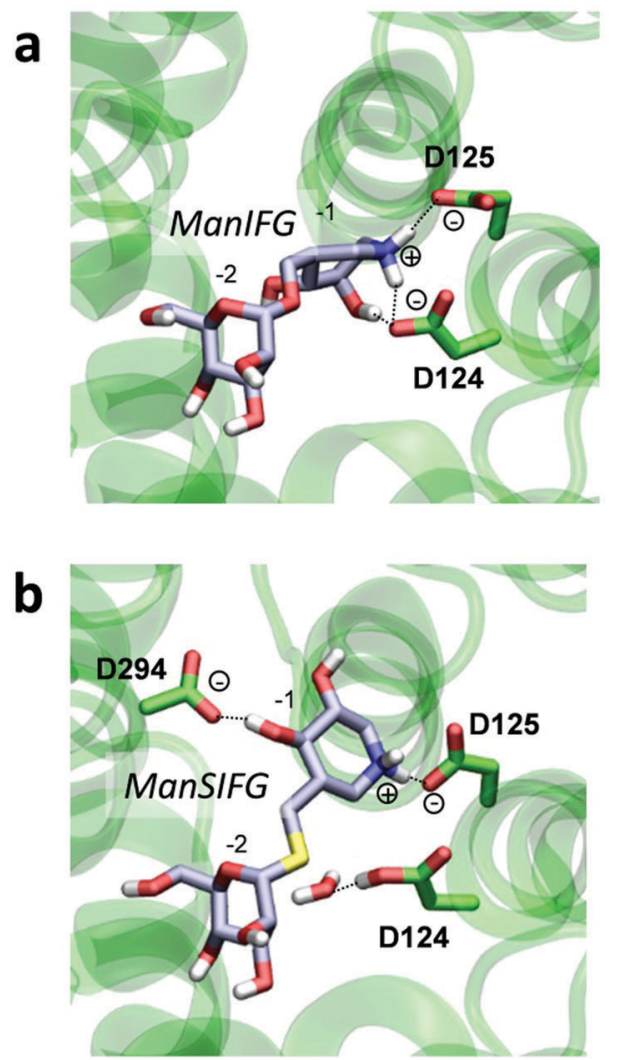

Fig. 3 Calculated complexes of BcGH76 with (a) ManIFG 1 and (b) ManSIFG 2.

see also ESI, $\dagger$ Fig. S2, S3) could faithfully recapitulate the X-ray structures (for an overlay, see ESI, $\dagger$ Fig. S4). Therefore, there is change of protonation state of the catalytic residues upon binding of ManSIFG in comparison to ManIFG, concurrent with the change of binding pose.

Additional simulations in which $\mathrm{O}$ was replaced by $\mathrm{S}$ (and vice versa) in the corresponding complexes did not result in reversion of the binding mode of the ligand (i.e. replacement of $\mathrm{O}$ by $\mathrm{S}$ in $B c$ GH76-ManIFG did not make the in silico complex evolve towards the "observed" $B c$ GH76-ManSIFG, and vice versa). This suggests that the individual binding poses are stable states separated by a sizable energy barrier and that the two ligands achieve their respective modes directly during binding and not by inter-conversion between binding modes once bound. Further, for the $B c$ GH76-ManIFG complex (Fig. 3a), the nucleophile-ammonium interaction appears to dominate binding, while the protonation state of the acid/base (D125) is less important, most likely because of the lack of an aglycon. By contrast, for the $B c$ GH76-ManSIFG complex (Fig. 3b), in which the inhibitor ammonium group interacts with the deprotonated acid/base residue (see ESI, $\uparrow$ Fig. S5), the nucleophile (D124) must be protonated. In this case, both experimentally and throughout the MD simulation, a water molecule is observed to interact with the protonated nucleophile that approximately occupies the position of the 3-OH residue in the ManIFG complex, thereby recapitulating this interaction.

S-Linked oligosaccharides are useful substrate mimics that have been used to illuminate the active site details of a range of 
glycosidases; ${ }^{26}$ however, their use has not been without controversy. For example, recent work on a GH family $125 \alpha$-mannosidase from Clostridium perfringens ( $C p \mathrm{GH} 125$ ) revealed significant differences in the preferred conformations of $\mathrm{O}$ - and S-linked 1,6- $\alpha$-mannobioses bound in the $-1 /+1$ subsites. $^{27}$ Thus, while the S-linked 1,6- $\alpha$ thiomannobiose was observed to bind in a ${ }^{4} C_{1}$ conformation, QM/MM MD predicted the 1,6- $\alpha$-mannobiose to bind in an ${ }^{\mathrm{O}} S_{2}$ conformation to wildtype, and X-ray crystallography revealed it to bind in the same conformation to a disabled enzyme mutated at the catalytic acid residue. Another notable example is that of an active-site spanning Michaelis complex of a thiocellopentaoside bound to a Fusarium oxysporum cellulase, for which electron density for only three sugar residues could be observed in the X-ray structure. ${ }^{28}$ In both cases these anomalous observations likely result from the longer length of a C-S bond (1.89 vs. $1.48 \AA$ for $\mathrm{C}-\mathrm{O}$ ) in a thioglycoside. ${ }^{27}$ These two prior studies, and the present work bring to attention the subtle yet significant limitations that sulfur-for-oxygen substitution within glycosides can have upon biological recognition by glycosidases.

We are supported by the Royal Society (Ken Murray Research professorship to G. J. D.), the European Research Council (ERC-2012-AdG-32294 "Glycopoise" to G. J. D. and Y. J.), the Australian Research Council (FT130100103) (S. J. W.), the Spanish Ministry of Economy, Industry and Competitiveness (MINECO, CTQ2014-55174-P) (C. R. and J. C.) and Generalitat de Catalunya-AGAUR (2014SGR-987) (C. R.). We thank Diamond Light Source for access to beamline I02 and I04 (mx-13587), BSC-CNS for computer resources and technical support at MareNostrum (RES-QCM-2016-3-0017), and Amicus Therapeutics. J. C. thanks MINECO for a PhD scholarship (FPI).

\section{Notes and references}

1 S. J. Free, Adv. Genet., 2013, 81, 33-82.

2 M. Jankute, J. A. Cox, J. Harrison and G. S. Besra, Annu. Rev. Microbiol., 2015, 69, 405-423.

3 J. Ruiz-Herrera, M. V. Elorza, E. Valentin and R. Sentandreu, FEMS Yeast Res., 2006, 6, 14-29.

4 B. Cao and S. J. Williams, Nat. Prod. Rep., 2010, 27, 919-947.

5 V. Lombard, H. Golaconda Ramulu, E. Drula, P. M. Coutinho and B. Henrissat, Nucleic Acids Res., 2014, 42, D490-D495.

6 H. Kitagaki, H. Wu, H. Shimoi and K. Ito, Mol. Microbiol., 2002, 46, 1011-1022; J. Ao, J. L. Chinnici, A. Maddi and S. J. Free, Eukaryotic Cell, 2015, 14, 792-803; H. Kitagaki, K. Ito and H. Shimoi, Eukaryotic Cell, 2004, 3, 1297-1306; E. Spreghini, D. A. Davis, R. Subaran, M. Kim and A. P. Mitchell, Eukaryotic Cell, 2003, 2, 746-755.
7 F. Cuskin, E. C. Lowe, M. J. Temple, Y. Zhu, E. A. Cameron, N. A. Pudlo, N. T. Porter, K. Urs, A. J. Thompson, A. Cartmell, A. Rogowski, B. S. Hamilton, R. Chen, T. J. Tolbert, K. Piens, D. Bracke, W. Vervecken, Z. Hakki, G. Speciale, J. L. Munoz-Munoz, A. Day, M. J. Pena, R. McLean, M. D. Suits, A. B. Boraston, T. Atherly, C. J. Ziemer, S. J. Williams, G. J. Davies, D. W. Abbott, E. C. Martens and H. J. Gilbert, Nature, 2015, 517, 165-169.

8 T. Nakajima, S. K. Maitra and C. E. Ballou, J. Biol. Chem., 1976, 251, 174-181; Y. Maruyama and T. Nakajima, Biosci., Biotechnol., Biochem., 2000, 64, 2018-2020.

9 A. J. Thompson, G. Speciale, J. Iglesias-Fernandez, Z. Hakki, T. Belz, A. Cartmell, R. J. Spears, E. Chandler, M. J. Temple, J. Stepper, H. J. Gilbert, C. Rovira, S. J. Williams and G. J. Davies, Angew. Chem., Int. Ed., 2015, 54, 5378-5382.

10 T. M. Gloster and D. J. Vocadlo, Nat. Chem. Biol., 2012, 8, 683-694; V. H. Lillelund, H. H. Jensen, X. Liang and M. Bols, Chem. Rev., 2002, 102, 515-553; B. Ganem, Acc. Chem. Res., 1996, 29, 340-347; T. D. Heightman and A. T. Vasella, Angew. Chem., Int. Ed., 1999, 38, 750-770.

11 S. Moréra, A. Vigouroux and K. A. Stubbs, Org. Biomol. Chem., 2011, 9, 5945-5947.

12 C. Li, A. Begum, S. Numao, K. H. Park, S. G. Withers and G. D. Brayer, Biochemistry, 2005, 44, 3347-3357.

13 O. A. Andersen, M. J. Dixon, I. M. Eggleston and D. M. van Aalten, Nat. Prod. Rep., 2005, 22, 563-579.

14 W. M. Best, V. Ferro, J. Harle, R. V. Stick and D. M. G. Tilbrook, Aust. J. Chem., 1997, 50, 463-472.

15 W. Dong, T. Jespersen, M. Bols, T. Skrydstrup and M. R. Sierks, Biochemistry, 1996, 35, 2788-2795.

16 J. M. Macdonald, C. A. Tarling, E. J. Taylor, R. J. Dennis, D. S. Myers, S. Knapp, G. J. Davies and S. G. Withers, Angew. Chem., Int. Ed., 2010, 49, 2599-2602.

17 T. Belz and S. J. Williams, Carbohydr. Res., 2016, 429, 38-47.

18 P. J. Garegg and B. Samuelsson, J. Chem. Soc., Perkin Trans. 1, 1980, 2866.

19 K. L. Matta, R. N. Girotra and J. J. Barlow, Carbohydr. Res., 1975, 43, 101-109.

20 F. M. Ibatullin, S. I. Selivanov and A. G. Shavva, Synthesis, 2001, 419-422.

21 M. von Itzstein, W.-Y. Wua and B. Jina, Carbohydr. Res., 1994, 259, 301-305.

22 R. J. Williams, J. Iglesias-Fernandez, J. Stepper, A. Jackson, A. J. Thompson, E. C. Lowe, J. M. White, H. J. Gilbert, C. Rovira, G. J. Davies and S. J. Williams, Angew. Chem., Int. Ed., 2014, 53, 1087-1091.

23 M. D. Winn, C. C. Ballard, K. D. Cowtan, E. J. Dodson, P. Emsley, P. R. Evans, R. M. Keegan, E. B. Krissinel, A. G. W. Leslie, A. McCoy, S. J. McNicholas, G. N. Murshudov, N. S. Pannu, E. A. Potterton, H. R. Powell, R. J. Read, A. Vagin and K. S. Wilson, Acta Crystallogr., Sect. D: Struct. Biol., 2011, 67, 235-242.

24 A. Barducci, M. Bonomi and M. Parrinello, Wiley Interdiscip. Rev.: Comput. Mol. Sci., 2011, 1, 826-843; A. Laio and M. Parrinello, Proc. Natl. Acad. Sci. U. S. A., 2002, 99, 12562-12566.

25 A. Varrot, C. A. Tarling, J. M. Macdonald, R. V. Stick, D. L. Zechel, S. G. Withers and G. J. Davies, J. Am. Chem. Soc., 2003, 125, 7496-7497. 26 H. Driguez, ChemBioChem, 2001, 2, 311-318.

27 S. Alonso-Gil, A. Males, P. Z. Fernandes, S. J. Williams, G. J. Davies and C. Rovira, J. Am. Chem. Soc., 2017, 139, 1085-1088.

28 G. Sulzenbacher, H. Driguez, B. Henrissat, M. Schulein and G. J. Davies, Biochemistry, 1996, 35, 15280-15287. 